\title{
The Relationship of Tuning and Noise Correlations in Macaque Auditory Cortex
}

\author{
-Elena Andreeva \\ Neuroscience Center Zurich International PhD Program in Neuroscience, Institute of Neuroinformatics, University of Zürich and Eidgenössische \\ Technische Hochschule Zürich, 8057 Zürich, Switzerland \\ Review of Downer et al.
}

All neurons are noisy, but some neurons are more noisy than others, Downer and colleagues (2015) report in a recent issue of The Journal of Neuroscience. Sources of biological noise are plenty: from the stochastic nature of presynaptic neurotransmitter release and postsynapticion channel opening to the ongoing membrane voltage fluctuations arising from oscillatory network dynamics, a single neuron's response varies with each presentation of a stimulus. Nonetheless, the brain achieves a remarkably stable and invariant representation of the external world.

One computational strategy to overcome noise is to average it out by pooling responses of many cells. A caveat, however, is that neurons (especially neighbors) often covary in their stimulus-unrelated firing rate fluctuations. Unlike independent noise, such positively-correlated fluctuations cannot be cancelled by averaging, since in neurons with similar tuning, a joint peak in activity due to shared noise cannot be distinguished from a peak in activity due to stimulus drive. If neurons are anti-correlated in their stimulus response, however, the reverse holds

Received July 13, 2015; revised Aug. 22, 2015; accepted Aug. 24, 2015.

I thank Kevan Martin, Sepp Kollmorgen, and Saray Soldado Magraner for helpful discussion, manuscript proofreading, and indispensable moral support.

Correspondence should be addressed to Elena Andreeva, Institute of Neuroinformatics, University of Zürich, Winterthurerstrasse 190, 8057 Zürich, Switzerland. E-mail: elena@ini.ethz.ch.

DOI:10.1523/JNEUROSCI.2665-15.2015

Copyright $\odot 2015$ the authors $\quad 0270-6474 / 15 / 3512974-03 \$ 15.00 / 0$ true: shared noise can be eliminated and the signal response concomitantly strengthened through subtractive pooling. Thus, information carried in the neural code is maximized for pairs of cells that have opposite signs of their signal and noise correlations (Averbeck et al., 2006).

Downer and colleagues (2015) present evidence for correlation-based coding optimization in the primate brain. The authors set out to examine the changes in the signal and noise correlation structure that occur within the primary auditory cortex (A1) of macaques as the behavioral relevance of the auditory input is varied. Specifically, they asked: does the transition from passive listening to active engagement in an auditory task increase or decrease noise correlation between pairs of cells in A1? Are pairs with similar tuning affected in the same way as dissimilarly tuned pairs, and does the overall effect benefit or compromise stimulus discriminability in A1?

Three rhesus macaques (Macaca mulatta) were trained to detect amplitude modulation (AM) of sound. In a $\mathrm{Go} /$ No-Go task, the monkeys compared two successive broadband noise stimuli: an unmodulated "standard" stimulus followed by a "test," which could either be unmodulated or convolved with a sinusoidal AM envelope. When modulation occurred, the animals had to release a bar within $800 \mathrm{~ms}$ of sound offset to receive a reward.

Activity was recorded simultaneously from pairs of cells in A1 as the animals performed the task (active blocks) or heard the same stimuli without being required to respond (passive blocks). For each pair, tuning similarity ( $\mathrm{r}_{\text {tuning }}$ ) was quantified by deriving the Pearson correlation coefficient of the cells' mean responses at different AM depths. Given inherent variability of neurons, a cell's response will deviate from its mean on any given trial. For a pair of cells, activity fluctuations over trial repetitions may rise and fall in concert-that is, the two cells may share noise. The Pearson correlation of their trial-by-trial responses gives a measure of each pair's mutual noise ( $\left.r_{\text {noise }}\right)$.

The authors examined the population $r_{\text {tuning }}$ and $r_{\text {noise }}$ distributions as the animals transitioned between the passive listening and engaged states. On average, task engagement led to a decrease in noise correlations across A1, an effect previously observed with spatial and feature-selective attention in the primate visual system (Cohen and Maunsell, 2009; Mitchell et al., 2009). Curiously, Cohen and Maunsell (2009) saw a homogenous decrease of pairwise noise correlations across visual area V4 regardless of the cells' tuning similarity. In contrast, Downer and colleagues (2015) found lower values of $r_{\text {noise }}$ only for cell pairs positively correlated in their stimu- 
lus response, with no difference observed for pairs with negative $r_{\text {tuning }}$ [removing shared noise from which would have reduced information in the population code (Averbeck et al., 2006)]. Thus, auditory task engagement appears to have only eliminated noise deleterious to A1 stimulus discriminability.

In fact, for optimal decoding, $r_{\text {noise }}$ of dissimilarly tuned cells would even be expected to increase. Jeanne et al. (2013) observed this effect in a higher-order auditory brain area of songbirds trained in a song motif discrimination task. Comparing neural responses to task-relevant and -irrelevant motifs, the authors found the theoretically optimal negative relationship between signal and noise correlations exclusively for motifs that the birds learned to use for solving the task. Given that this correlation structure persisted under anesthesia, it likely reflected a more permanent reorganization of network connectivity, for which there may not be enough time when a monkey transitions from pasive to engaged listening. Even without the information-maximizing increase in $r_{\text {noise }}$ for cells with negative $r_{\text {tuning }}$, Downer et al. (2015) found that the correlation distribution between pairs of cells in the engaged condition improved performance of a binary classifier in discriminating the amplitude-modulated stimuli that the monkeys had to detect. Thus, even on the timescale of a single behavioral session, stimulus decoding within A1 can adjust to the cognitive goals of the animal, enabling better readout of task-relevant stimuli.

Not all forms of learning-induced changes in auditory cortex correlation structure have shown concomitant enhancement of stimulus decoding. Discriminability of pup calls in mouse A1, for example, does not differ between virgin and mother mice, despite an almost twofold increase in noise correlations of the latter (Rothschild et al., 2013). Because stimuli in that study were classified using complete neural ensemble activity, unlike in the studies by Downer et al. (2015) and Jeanne et al. (2013), who based classification on cell pair responses, the relationship between tuning and noise correlations was not accounted for. It may very well be that the increase in shared noise found in mothers improved discriminability in pairs with negative $r_{\text {tuning }}$ while degrading it in pairs with positive $r_{\text {tuning, }}$, so that on the population level, the overall decoding effectiveness remained the same.
While common feedforward input is believed to be the main source of shared noise among neurons, activity cofluctuations may also arise from recurrent dynamics of local networks. In layer $2 / 3$ of mouse primary visual cortex (V1), cells with direct synaptic connections tend to exhibit higher correlation levels in both signal and noise. Correlations arising from recurrent excitation within subnetworks of cells with similar tuning could benefit feature extraction by making the population response more robust against single-cell variability (Ko et al., 2011).

In primary sensory areas, correlations may have a distinct functional role when induced by feedback from higher processing regions. Studying object contour assignment in V1 and V2 of behaving macaques, Martin and von der Heydt (2015) showed that cells with consistent border-ownership preferences synchronized their spiking when edges of a common object fell into their receptive fields (the synchrony was lost when the same edges belonged to separate objects). The stronger the synchrony, the faster the monkeys reacted. The authors proposed that this synchronization reflects feedback drive from feature-binding "grouping cells," but gave no suggestion as to where such cells might reside.

A hint comes from Pooresmaeili et al. (2014). Measuring noise correlations between cells in the frontal eye field and V1 of macaques carrying out a curvetracing task, they found stronger coherence between the two areas during attention. Since an object's contour necessarily engages cells with different orientation preferences, such top-down grouping could synchronize activity of cells with negative $r_{\text {tuning }}$. What may appear as "noise" in experiments using simple stimuli may actually reflect higher-level network dynamics. In light of population coding theory, it follows that low-level features would become more differentiable when cells encoding them are correlated through feedback from higher processing centers.

Thus, noise correlations may be a signature of functional subnetworks that transiently emerge in cortex in accordance with changing behavioral goals of the animal. The first evidence for behaviorally driven changes in correlation structure of monkey auditory cortex was found over 20 years ago by Ahissar et al. (1992) in an ingenious experiment relating attention to spike-timing-dependent plasticity. In a cellular conditioning protocol, the spiking of one cell would trigger the presentation of an auditory stimulus tailored to elicit a response in a second simultaneously recorded cell. Throughout the conditioning process, the monkeys monitored the spike-triggered sounds for a change in frequency.

Like Downer and colleagues (2015), Ahissar et al. (1992) compared the relative impact that task engagement and passive listening had on the recorded pairs' activity correlations. Though conditioning increased the cells' spiking contingencies regardless of behavior, potentiation was much stronger when the monkeys attended the sounds to perform the task. The increased correlation between the spike trains decayed over several minutes of spontaneous activity, consistent with Downer et al.'s (2015) idea that behaviorally induced changes in auditory cortex are both rapid and flexible. Attention has been shown to modify the receptive fields of neurons in ferret $\mathrm{A} 1$ on a similar timescale (Fritz et al, 2003).

One glossed-over but perhaps worrying fact is that attention and learningrelated changes in noise correlation magnitude tend to be small, raising the question of their relative significance for cortical stimulus processing. Noise may be important, but for reasons altogether different than optimal population coding. Work in artificial intelligence, for example, has demonstrated that a degree of stochastic silencing of units during learning can greatly aid neural network performance (Hinton et al., 2012). Downer and colleagues (2015) have shown the importance of characterizing noise in the context of cellular tuning rather than treating it as a blanket population phenomenon. Future work should reveal whether stimulus-independent covariations in neural activity are indeed detrimental to coding or, alternatively, reveal stamps of a higher-order architectural plan, substructures of which are dynamically brought in and out of focus to accommodate the demands of the task at hand.

\section{References}

Ahissar E, Vaadia E, Ahissar M, Bergman H, Arieli A, Abeles M (1992) Dependence of cortical plasticity on correlated activity of single neurons and on behavioral context. Science 257: 1412-1415. CrossRef Medline

Averbeck BB, Latham PE, Pouget A (2006) Neural correlations, population coding and computation. Nat Rev Neurosci 7:358-366. CrossRef Medline

Cohen MR, Maunsell JH (2009) Attention improves performance primarily by reducing interneuronal correlations. Nat Neurosci 12: 1594-1600. CrossRef Medline

Downer JD, Niwa M, Sutter ML (2015) Task engagement selectively modulates neural 
correlations in primary auditory cortex. J Neurosci 35:7565-7574. CrossRef Medline

Fritz J, Shamma S, Elhilali M, Klein D (2003) Rapid task-related plasticity of spectrotemporal receptive fields in primary auditory cortex. Nat Neurosci 6:1216-1223. CrossRef Medline

Hinton GE, Srivastave N, Krizhevsky A, Sutskever I, Salakhutdinov RR (2012) Improving neural networks by preventing co-adaptation of feature detectors. arXiv:1207.0580.

Jeanne JM, Sharpee TO, Gentner TQ (2013) Associative learning enhances population cod- ing by inverting interneuronal correlation patterns. Neuron 78:352-363. CrossRef Medline

Ko H, Hofer SB, Pichler B, Buchanan KA, Sjöström PJ, Mrsic-Flogel TD (2011) Functional specificity of local synaptic connections in neocortical networks. Nature 473:87-91. CrossRef Medline

Martin AB, von der Heydt R (2015) Spike synchrony reveals emergence of proto-objects in visual cortex. J Neurosci 35:6860-6870. CrossRef Medline

Mitchell JF, Sundberg KA, Reynolds JH (2009)
Spatial attention decorrelates intrinsic activity fluctuations in macaque area $\mathrm{V} 4$. Neuron 63:879-888. CrossRef Medline

Pooresmaeili A, Poort J, Roelfsema PR (2014) Simultaneous selection by object-based attention in visual and frontal cortex. Proc Natl Acad Sci U S A 111:6467-6472. CrossRef Medline

Rothschild G, Cohen L, Mizrahi A, Nelken I (2013) Elevated correlations in neuronal ensembles of mouse auditory cortex following parturition. J Neurosci 33:12851-12861. CrossRef Medline 